\title{
Abortion and mental health: quantitative synthesis and analysis of research published 1995-2009
}

\author{
Priscilla K. Coleman
}

\section{Background}

Given the methodological limitations of recently published qualitative reviews of abortion and mental health, a quantitative synthesis was deemed necessary to represent more accurately the published literature and to provide clarity to clinicians.
Aims
To measure the association between abortion and indicators of adverse mental health, with subgroup effects calculated based on comparison groups (no abortion, unintended pregnancy delivered, pregnancy delivered) and particular outcomes. A secondary objective was to calculate population-attributable risk (PAR) statistics for each outcome

\section{Method}
After the application of methodologically based selection criteria and extraction rules to minimise bias, the sample comprised 22 studies, 36 measures of effect and 877181 participants (163831 experienced an abortion). Random effects pooled odds ratios were computed using adjusted odds ratios from the original studies and PAR statistics were derived from the pooled odds ratios.

\section{Results}

Women who had undergone an abortion experienced an $81 \%$ increased risk of mental health problems, and nearly $10 \%$ of the incidence of mental health problems was shown to be attributable to abortion. The strongest subgroup estimates of increased risk occurred when abortion was compared with term pregnancy and when the outcomes pertained to substance use and suicidal behaviour.

\section{Conclusions}

This review offers the largest quantitative estimate of mental health risks associated with abortion available in the world literature. Calling into question the conclusions from traditional reviews, the results revealed a moderate to highly increased risk of mental health problems after abortion. Consistent with the tenets of evidence-based medicine, this information should inform the delivery of abortion services.

\section{Declaration of interest}

None.

Second, many recently published studies with extensive controls for third variables were not reflected in the three recent reviews, with no explanation given as to why large segments of the peer-reviewed literature were missing. For instance, in the 2008 review by Charles et $a l^{6}{ }^{6}$ several of the studies that were overlooked actually met the inclusion criteria. ${ }^{10-19}$ Similarly, studies examining substance misuse were not included in two of the three reviews, ${ }^{6,7}$ with no rationale for excluding them. Numerous studies have demonstrated statistically significant associations between abortion and subsequent substance misuse, a widely recognised and prevalent mental health problem. ${ }^{2,10,20-24}$

Third, in all three literature reviews the choice of studies lacked sufficient methodologically based selection criteria. ${ }^{5-7}$ As a result the sample of studies included was either too broad, resulting in incorporation of results from numerous weaker studies, or too narrow, resulting in unjustified elimination of sound studies. Ironically, the largest review, by the American Psychological Association Task Force, exemplifies both problems as the selection criteria for one type of study (those with a comparison group) were simply publication of empirical data on induced abortion with at least one mental health measure in peer-reviewed journals in English on US and non-US samples; ${ }^{5}$ however, non-US samples were avoided entirely for a second type of study (no comparison group) examined in this review without an appropriate rationale, resulting in elimination of dozens of methodologically sophisticated international studies. In the review conducted by Robinson et al the authors mention having identified 216 peer-reviewed papers on the topic of abortion and mental health and then note selection of a sample of studies that 'exemplify common errors in research methodology' as well as 'major articles that attempt to correct the flaws.' were offered regarding how studies were chosen to fit into these two categories. 
The fourth troubling issue is the fact that quantification of effects was not attempted by any of the three research teams. Given the expansive literature on abortion and mental health, there is no reasonable justification for not quantifying effects. In the only truly systematic review available, published in 2003 by Thorp et al, stringent selection criteria were employed and their analysis of the largest and strongest studies available resulted in the conclusion that abortion is associated with an increased risk of depression that may lead to self-harm. ${ }^{4}$ Owing to the broad objective of this review, which addressed physical complications as well, a wide range of mental health effects were not examined.

In this highly politicised area of research it is imperative for researchers to apply scientifically based evaluation standards in a systematic, unbiased manner when synthesising and critiquing research findings. If not, authors open themselves up to accusations of shifting standards based on conclusions aligned with a particular political viewpoint. Moreover, the results may be dangerously misleading and result in misinformation guiding the practice of abortion. Through a process of systematically combining the quantitative results from numerous studies addressing the same basic question (e.g. 'is there an association between abortion and mental health?') far more reliable results are produced than from particular studies that are limited in size and scope. Moreover, as a methodology wherein studies are weighted based on objective scientific criteria, meta-analysis offers a logical, more objective alternative to qualitative reviews when the area of study is embedded in political controversy. Therefore, in an effort to provide a long overdue, dispassionate analysis of the literature on abortion and mental health, the primary objective of this review was to conduct meta-analyses of associations between induced abortion and adverse mental health outcomes (depression, anxiety, substance use and suicidal behaviour) with sensitivity to the use of distinct control groups employed in the various studies (no abortion, unintended pregnancy delivered, pregnancy delivered). The focus was on studies published between 1995 and 2009 because of the considerable improvement in research designs on the topic of post-abortion mental health in recent years. Contemporary research on abortion and mental health has addressed a number of shortcomings of the earlier work by employing comparison groups with controls for third variables. However, there has also been increased emphasis on incorporating nationally representative samples, prospective designs, controls for prior psychiatric history and comprehensive assessments of mental health outcome measures which in some cases included actual medical records. A secondary objective of this review was to calculate population-attributable risk (PAR) percentages using pooled odds ratios derived from the meta-analysis subdivided by outcome measures. These statistics reflect the incidence of a disorder in the exposed sample (e.g. women who have undergone abortion) that is directly due to the exposure (the abortion procedure). Both the pooled odds ratios and the PAR percentages yielded herein provide readily interpretable indices of the mental health consequences of abortion and should offer new clarity to the academic debate and to clinicians seeking information to guide effective practice.

\section{Method}

\section{Inclusion criteria}

Studies identified using the Medline and PsycINFO databases were included in this review if they met the following criteria: a sample size of 100 or more participants; use of a comparison group (no abortion, pregnancy delivered or unintended pregnancy delivered); one or more mental health outcome variables (depression, anxiety, alcohol use, marijuana use or suicidal behaviour); controls for third variables; use of odds ratios to express effects observed to facilitate calculation of readily interpretable pooled odds ratios and PAR statistics; publication in English in peer-reviewed journals between 1995 and 2009.

\section{Rules for extraction and synthesis of effects}

In addition to the above criteria, rules for extracting and synthesising data derived from the studies selected were developed based on the recommendations outlined by Lipsey, ${ }^{25}$ to avoid overrepresentation of particular samples and statistical dependences among effects, and generally to ensure the most conservative and unbiased assemblage of results from the individual studies exhibiting considerable variability in reporting.

(a) Relevant studies contributed a maximum of one effect per outcome. When authors reported more than one effect per variable based on separate analyses conducted for distinct demographic groups, or when different diagnoses were reported on within a general class such as anxiety or depression, a composite odds ratio was derived to avoid overweighting in favour of particular studies.

(b) When studies had more than one comparison group, selection rules were employed to provide more weight to comparisons wherein the control group was most closely matched to the abortion group. Specifically, if 'unintended pregnancy delivered' was used the results relative to this group were selected, and when only 'pregnancy delivered' and 'no abortion' comparison groups were used, the effects pertaining to the 'pregnancy delivered' group were selected.

(c) In situations wherein separate results were reported based on one $v$. two or more abortions, the results specific to one abortion were selected to enable sampling of a more homogeneous population. There are studies suggesting differential effects based on the number of abortions. ${ }^{26,27}$

(d) When particular authors used the same sample and variables in more than one publication, only the most recent publication was selected. When the same data-set was used by different groups, both sets of results were included when distinct samples were defined.

\section{Statistical analysis}

Meta-analyses were conducted using Comprehensive Meta-Analysis version 2.0 for Windows (Biostat, www.meta-analysis.com). Random effects meta-analyses were computed based on the sociodemographic heterogeneity of the study samples. ${ }^{43}$ The random effects model takes into account two sources of variance (within-study error and variation in the true effects across studies) with the study weights designed to minimise both sources of variance. ${ }^{43}$ A pooled odds ratio was computed using the full 36 effects extracted. In addition, two sets of subgroup pooled odds ratios were calculated based on the type of comparison group used and on specific forms of mental health problems. Adjusted odds ratios with controls for third variables were used in all the random effects meta-analyses. Finally, PAR percentages were computed using the pooled odds ratios (OR) derived from the random effects model subdivided by outcome measures. The PAR percentages were calculated using the formula $100 \times(P x(\mathrm{OR}-1)) /$ $(1+P x(\mathrm{OR}-1))$, where $P x$ is the estimate of population exposure; $P x$ is calculated as $c /(c+d)$, where $c$ is the number of women in the abortion group who did not experience the mental illness in question and $d$ is the number of women in the 'no abortion' group who were identified as not having the mental illness examined. 


\section{Results}

After applying the inclusion criteria and rules detailed above, the sample consisted of 22 peer-reviewed studies ( 15 from the USA and 7 from other countries); ;,20-22,24,26-42 these comprised 36 measures of effect ( 9 alcohol use/misuse, 5 marijuana, 7 anxiety, 11 depression, 4 suicidal behaviour) and a total of 877181 participants, of whom 163831 had experienced an abortion (see online Table DS1).

The first random effects meta-analysis, which included 36 adjusted odds ratios from the 22 studies identified, resulted in a pooled odds ratio of 1.81 (95\% CI 1.57-2.09, $P<0.0001$ ). The results of this analysis indicated that women who have had an abortion experienced an $81 \%$ higher risk of mental health problems of various forms when compared with women who had not had an abortion (Fig. 1). Results of a second random effects meta-analysis, wherein separate effects were produced based on the type of outcome measure, are provided in Fig. 2. All effects were statistically significant, with the largest pooled odds ratio derived for marijuana use $(\mathrm{OR}=3.30,95 \% \mathrm{CI} 1.64$ 7.44, $P=0.001)$, followed by suicide behaviours $(\mathrm{OR}=2.55,95 \%$ CI 1.31-4.96, $P=0.006)$, alcohol use/misuse $(\mathrm{OR}=2.10,95 \% \mathrm{CI}$ $1.77-2.49, P<0.0001)$, depression $(\mathrm{OR}=1.37,95 \%$ CI 1.22 $1.53, P<0.0001)$ and anxiety $(\mathrm{OR}=1.34,95 \%$ CI $1.12-1.59$, $P<0.0001)$. These results indicate that the level of increased risk associated with abortion varies from $34 \%$ to $230 \%$ depending on the nature of the outcome.

In the third random effects meta-analysis (Fig. 3) three separate pooled odds ratios were produced based on the type of comparison group employed in the respective studies. When women who had terminated a pregnancy were compared with women who had not done so relative to all mental health problems, the result was statistically significant $(\mathrm{OR}=1.59,95 \%$ CI 1.36-1.85, $P<0.0001)$. When women who terminated a pregnancy were compared with women who carried to term, using the full set of mental health variables, the result was considerably stronger $(\mathrm{OR}=2.38,95 \% \mathrm{CI} 1.62-3.50, P<0.0001)$. Finally, when 'unintended pregnancy carried to term' operated as the comparison group, the result was likewise statistically significant and closer to the result relative to the 'no abortion' comparison group $(\mathrm{OR}=1.55,95 \% \mathrm{CI} 1.30-1.83, P<0.0001)$. These data indicate that regardless of the type of comparison group used, abortion is associated with an enhanced risk of experiencing mental health problems, with the magnitude of this risk ranging from $55 \%$ to $138 \%$.

The last set of analyses involved calculation of PAR percentages based on pooled odds ratio estimates. The overall PAR percentage was nearly $10 \%$, with the range for particular mental health problems extending from $8.3 \%$ for anxiety to $26.5 \%$ for marijuana use (Table 1 ). In addition, a pooled odds ratio for the two large-scale studies in which actual suicide was
Study name

Coleman $2006^{10}$ alco

Coleman $2006^{10}$ marij

Coleman et al $2009^{20}$ alco

coleman et al $2009^{20}$ anx

Coleman et al $2009^{20}$ dep

coleman et al $2009^{28}$ alco

coleman et al $2005^{26}$ alco

Coleman et al $2002^{29}$ alco

Coleman et al $2002^{29}$ mari

coleman et al $2002^{30}$ anx

Coleman et al $2002^{30}$ dep

Cougle et al $2005^{32}$ anx

Cougle et al $2003^{32}$ dep

Dingle et al $2008^{21}$ dep

Dingle et al $2008^{21}$ alco

Dingle et al $2008^{21}$ anx

Dingle et al $2008^{21}$ marij

Fergusson et al $2008^{22}$ suic ideation

Fergusson et al $2008^{22}$ alco

Fergusson et al $2008^{22}$ anx

Fergusson et al $2008^{22}$ dep

Gilchrist et al $1995^{33}$ self-harm

Gissler et al $1996^{34}$ suic

Pederson $2007^{24}$ alco

Pederson $2007^{24}$ marij

Pederson $2008^{35}$ dep

Reardon \& Cougle $2002^{35}$ dep

Reardon et al $2004^{39}$ alco

Reardon et al $2004^{39}$ marij

Reardon et al $2003^{38}$ dep

Reardon et al $2002^{37}$ suic

Rees \& Sabia $2007^{40}$ dep

Schmiege \& Russo $2005^{41}$ dep

Steinberg \& Russo $2008^{27}$ anx/NCS

Steinberg \& Russo $2008^{27}$ anx/NCFG

Taft \& Watson $2008^{42}$ dep Total
Statistics for each study

\begin{tabular}{|c|c|c|c|c|}
\hline $\begin{array}{l}\text { ppper } \\
\text { limit }\end{array}$ & $\begin{array}{l}\text { Odds } \\
\text { ratio }\end{array}$ & $\begin{array}{l}\text { Lower } \\
\text { limit }\end{array}$ & z & $P$ \\
\hline 268 & 5.720 & 1.200 & 2.189 & 0.02 \\
\hline & & 1.990 & 2.854 & 0.004 \\
\hline & & & 4.014 & DOC \\
\hline & 1.7 & 1.360 & 4.171 & \\
\hline & & & 2.841 & \\
\hline & & 1.688 & 3.430 & 0.0 \\
\hline & & & 1773 & \\
\hline & & 02 & 4.609 & 0.000 \\
\hline$y$ & & & 8.814 & 0000 \\
\hline & & & 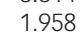 & .050 \\
\hline & & & 1.711 & \\
\hline & 1 & 1.053 & 2.38 & 0.017 \\
\hline & & & 2 & 0.013 \\
\hline & & & & \\
\hline & & & 2.9 & 0 \\
\hline & & & 1.6 & \\
\hline & & & & 0. \\
\hline & . & & 1.3 & 0.1 \\
\hline & & & & \\
\hline & & & 2.7 & 0. \\
\hline & & & & \\
\hline & 1.70 & 1.106 & 2.4 & 0.0 \\
\hline & & & 6.8 & \\
\hline & & & 2.1 & . \\
\hline & & & & \\
\hline & & & 0 & 0.3 \\
\hline & & & 16 & 810 \\
\hline & & & 1.7 & 0.0 \\
\hline & & 1.18 & 25 & (1) \\
\hline & & & 4.1 & 0.6 \\
\hline & & & 2.2 & . \\
\hline & & & 1.9 & 0.047 \\
\hline & & & 1.019 & \\
\hline & & & -0.4 & \\
\hline & & & & \\
\hline & & & & \\
\hline & & & & \\
\hline
\end{tabular}

Odds ratio and $95 \% \mathrm{Ci}$

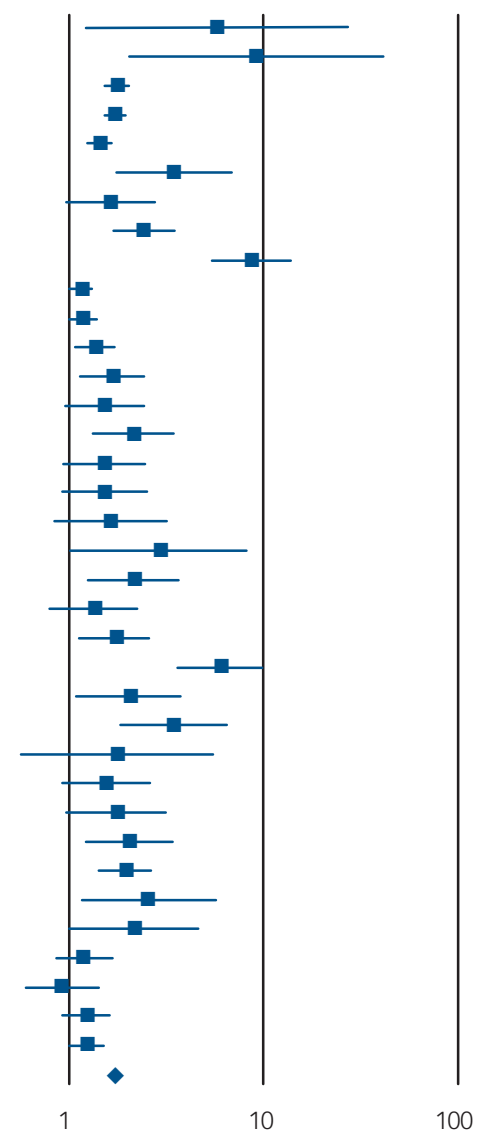

0.01
0.1

Favours no abortion

Favours abortion

Fig. 1 Abortion and subsequent mental health outcomes. alco, alcohol misuse; anx, anxiety; dep, depression; marij, marijuana use; NCS, National Comorbidity Survey; NCFG, National Survey of Family Growth; suic, suicide. 


\section{Alcohol}

Coleman $2006^{10}$

Coleman et al $2009^{20}$

Coleman et al $2009^{28}$

Coleman et al $2005^{26}$

Coleman et al $2002^{29}$

Dingle et al $2008^{21}$

Fergusson et al $2008^{22}$

Pederson $2007^{24}$

Reardon et al $2004^{39}$

All alcohol

Anxiety

Coleman et al $2009^{20}$

Coleman et al $2002^{30}$

Cougle et al $2005^{32}$

Dingle et al $2008^{21}$

Fergusson et al $2008^{22}$

Steinberg \& Russo $2008^{27}$ NCS

Steinberg \& Russo $2008^{27}$ NCFG

All anxiety

Depression

Coleman et al $2009^{20}$

Coleman et al $2002^{29}$

Cougle et al $2003^{31}$

Dingle et al $2008^{21}$

Fergusson et al $2008^{22}$

Pedersen $2008^{35}$

Reardon \& Cougle $2002^{36}$

Reardon et al $2003^{38}$

Rees \& Sabia $2007^{40}$

Schmiege \& Russo $2005^{41}$

Taft \& Watson $2008^{42}$

All depression

Marijuana use

Coleman $2006{ }^{10}$

Coleman et al $2002^{29}$

Dingle et al $2008^{21}$

Pedersen $2007^{24}$

Reardon et al $2004^{39}$

All marijuana use

Suicide and self-harm

Fergusson et al $2008^{22}$ suic ideation

Gilchrist et al $1995^{33}$ self-harm

Gissler et al $1996^{34}$ suic

Reardon et al $2002^{37}$ suic

All suicide/self-harm

\begin{tabular}{|c|c|c|c|c|}
\hline $\begin{array}{l}\text { oper } \\
\text { mit }\end{array}$ & $\begin{array}{l}\text { Odds } \\
\text { ratio }\end{array}$ & $\begin{array}{c}\text { Lower } \\
\text { limit }\end{array}$ & Z & $P$ \\
\hline 0 & & 1.200 & 2.189 & 0.029 \\
\hline & & 388 & 4.014 & 0.000 \\
\hline 810 & & 1.688 & 3.430 & 0.001 \\
\hline 761 & & 0.950 & 1.773 & 0.076 \\
\hline .474 & & 1.652 & 4.609 & 0.000 \\
\hline .446 & & 1.280 & 2.937 & 0.003 \\
\hline (n & & & 1.982 & 0.047 \\
\hline- & & 1.076 & 2.192 & 0.028 \\
\hline 3.112 & & 0.951 & 1.793 & \\
\hline & & 1.768 & 8.464 & 0.000 \\
\hline 2.34 & & 1.380 & 4.171 & 0.000 \\
\hline & & 1.000 & 1.958 & 0.050 \\
\hline & U & 1.053 & 2.381 & 0.017 \\
\hline & 0 & 0.919 & 1.620 & 0.105 \\
\hline & & 1.243 & 2.752 & 0.006 \\
\hline & & 0.588 & -0.400 & 0.689 \\
\hline & & 0.910 & 1.310 & 0.190 \\
\hline $5 \cap 0$ & 1340 & 1.123 & 3.253 & 0.001 \\
\hline & & 11 & 2.841 & 0.004 \\
\hline 1.37 & 0 & 0.979 & 1.711 & 0.087 \\
\hline 2.420 & 9 & 1.110 & 2.485 & 0.013 \\
\hline 449 & 0 & 0.919 & 1.620 & 0.105 \\
\hline 224 & 1. & 0.772 & 1.000 & 0.317 \\
\hline 484 & & 0.558 & 0.960 & 0.337 \\
\hline 2 & & 0. & 1. & 0. \\
\hline & & & 4.140 & 0.000 \\
\hline & 0 & 1.0 & 1.988 & 0.047 \\
\hline & & 0.8 & 1.019 & 0.308 \\
\hline & & 88 & 1.846 & 0.065 \\
\hline 1.535 & 370 & 1.223 & 5.421 & 0.000 \\
\hline & & 90 & 2.854 & 0.004 \\
\hline & 85 & 5 & 8.814 & 0.000 \\
\hline & & 0.900 & 1.556 & 0.120 \\
\hline & 3 & 1.803 & 3.782 & 0.000 \\
\hline & 20 & 1.180 & 2.575 & 0.010 \\
\hline An & & 1.649 & 3.261 & 0.001 \\
\hline & & & 1.377 & 0.168 \\
\hline & & & 2.418 & 0.016 \\
\hline & & & 6.878 & 0.000 \\
\hline & & & 2.278 & 0.023 \\
\hline & & & 2750 & \\
\hline
\end{tabular}

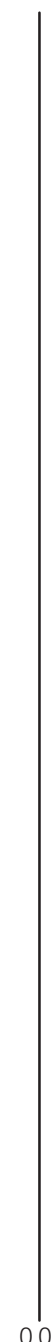

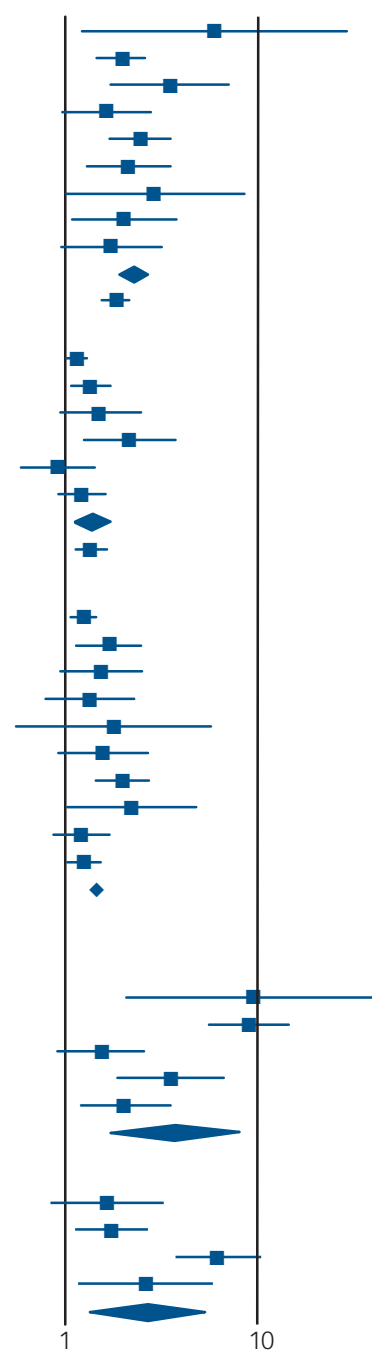

Favours abortion

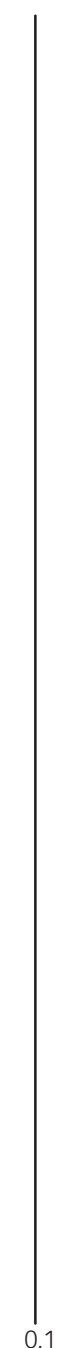

Favours no abortion

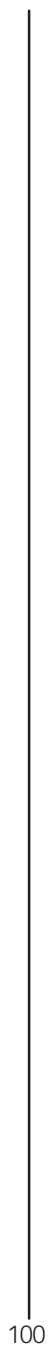

100

Fig. 2 Abortion and subsequent mental health outcomes, organised by dependent measures. NCS, National Comborbidity Survey; NCFG, National Survey of Family Growth; suic, suicide.

measured yielded a significant result $(\mathrm{OR}=4.11,95 \%$ CI 1.82 9.31) and a PAR percentage of $34.9 \%$ was derived using this pooled odds ratio.

\section{Discussion}

Based on data extracted from 22 studies, the results of this meta-analytic review of the abortion and mental health literature indicate quite consistently that abortion is associated with moderate to highly increased risks of psychological problems subsequent to the procedure. The magnitude of effects derived varied based on the comparison group (no abortion, pregnancy delivered, unintended pregnancy delivered) and the type of problem examined (alcohol use/misuse, marijuana use, anxiety, depression, suicidal behaviours). Overall, the results revealed that women who had undergone an abortion experienced an $81 \%$ increased risk of mental health problems, and nearly $10 \%$ of the incidence of mental health problems was shown to be directly attributable to abortion. The strongest effects were observed when women who had had an abortion were compared with women who had carried to term and when the outcomes measured related
Table 1 Population-attributable risk (PAR) percentages based on outcome measure

Outcome PAR \%

Anxiety

8.1

Depression

Alcohol use

Marijuana use

10.7

All suicidal behaviours

26.5

Suicide

All

20.9

34.9

9.9

to substance use and suicidal behaviour. Great care was taken to assess accurately the risks from the most methodologically sophisticated studies, and the quantitatively based conclusions reflect data gathered on over three-quarters of a million women. Of particular significance is the fact that all effects entered into the analyses were adjusted odds ratios with controls for numerous third variables.

The finding that abortion is associated with significantly higher risks of mental health problems compared with carrying 
Delivery

Coleman et al $2008^{28}$ alco

Coleman et al $2002^{29}$ alco

Coleman et al $2002^{29}$ marij

Coleman et al $2002^{30}$ anx

Coleman et al $2002^{30}$ dep

Cougle et al $2003^{31}$ dep

Gissler et al $1996^{34}$ suic

Pederson $2008^{35}$ dep

Reardon et al $2003^{38}$ dep

Reardon et al $2002^{37}$ suic

All delivery

No abortion

Coleman et al $2009^{20}$ alco

Coleman et al $2009^{20}$ anx

Coleman et al $2009^{20}$ dep

Coleman et al $2005^{26}$ alco

Dingle et al $2008^{21}$ dep

Dingle et al $2008^{21}$ alco

Dingle et al $2008^{21}$ anx

Dingle et al $2008^{21}$ marij

Pedersen $2007^{24}$ alc

Pedersen $2007^{24}$ marij

Rees \& Sabia $2007^{40}$ dep

Steinberg \& Russo $2008^{27}$ anx/NCS

Taft \& Watson $2008^{42}$ dep

All no abortion

Unintended pregnancy

Coleman $2006^{10}$ alco

Coleman $2006^{10}$ marij

Cougle et al $2005^{32}$ anx

Fergusson et al $2008^{22}$ suic ideation

Fergusson et al $2008^{22}$ alco

Fergusson et al $2008^{22}$ anx

Fergusson et al $2008^{22}$ dep

Gilchrist et al $1995^{33}$ self-harm

Reardon \& Cougle $2002^{36}$ dep

Reardon et al $2004^{39}$ alco

Reardon et al $2004^{39}$ marij

Schmiege \& Russo $2005^{41}$ dep

Steinberg \& Russo $2008^{27}$ anX/NCFG

All unintended pregnancy

\begin{tabular}{|c|c|c|c|c|}
\hline $\begin{array}{l}\text { pper } \\
\text { limit }\end{array}$ & $\begin{array}{l}\text { Odds } \\
\text { ratio }\end{array}$ & $\begin{array}{l}\text { Lower } \\
\text { limit }\end{array}$ & Z & $P$ \\
\hline 6.810 & 390 & 1.688 & 3.430 & . \\
\hline & & 1.652 & 4.609 & 0.000 \\
\hline .7 & & 5.307 & 8.814 & 0.000 \\
\hline 0 & & .000 & 1.958 & 0.050 \\
\hline 5 & & 0.979 & 1.711 & 0.087 \\
\hline & & 110 & 2.485 & 0.013 \\
\hline & & 58 & 6.878 & 0.000 \\
\hline 4 & & 0.568 & 0.960 & 0.337 \\
\hline 62 & & 1.411 & 4.140 & 0.000 \\
\hline 66 & & 1.139 & 2.278 & 0.023 \\
\hline 502 & & 1.626 & 4.443 & 0.000 \\
\hline & & 1.388 & 4.014 & 0.000 \\
\hline & & 1.380 & 4.171 & 0.000 \\
\hline 77 & 1.405 & 1.111 & 2.841 & 0.004 \\
\hline 76 & & 0.950 & 1.773 & 0.076 \\
\hline 2 & & 0.919 & 1.620 & 0.105 \\
\hline 42 & & & 2.937 & 0.002 \\
\hline 449 & 0 & 0.919 & 1.620 & 0.105 \\
\hline 0 & 0 & 0.900 & 1.556 & 0. \\
\hline 7 & 5 & 1.0 & 2.192 & 0.028 \\
\hline & & 1. & 3.782 & 0. \\
\hline & & 1. & 1.988 & 0.047 \\
\hline & & 0.588 & -0.400 & 0.689 \\
\hline & & 0.988 & 1.846 & 0.065 \\
\hline r & . & 1.366 & 5.939 & 0.000 \\
\hline & & 1.200 & 2.189 & 0.029 \\
\hline & & 1.990 & 2.854 & 0.004 \\
\hline & 1. & 1.0 & 2.381 & 0.017 \\
\hline & 1.610 & 0.818 & 1.377 & 0.168 \\
\hline & 2.880 & 1.012 & 1.982 & 0.047 \\
\hline 60 & & 1.243 & 2.752 & 0.006 \\
\hline & 1.310 & 0.772 & 1.000 & 0.317 \\
\hline 2 & 1.70 & 1.106 & 2.418 & 0.016 \\
\hline & & 0.90 & 1.606 & 0.108 \\
\hline & & 0.9 & 1.793 & 0.073 \\
\hline & & & 2.575 & 0.010 \\
\hline & & 0.852 & 1.019 & 0.308 \\
\hline & & 0.910 & 1.310 & 0.19 \\
\hline & & & & \\
\hline
\end{tabular}

0.1

Favours no abortion

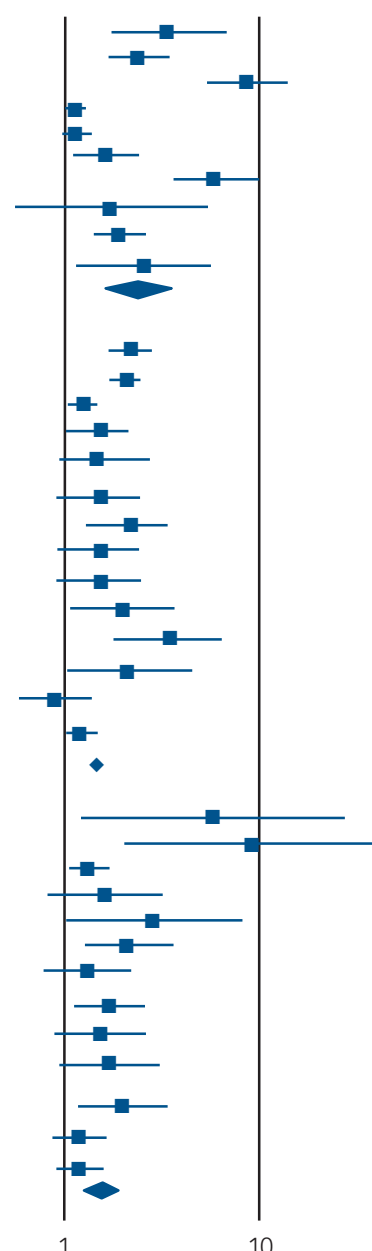

Favours abortion

Fig. 3 Abortion and subsequent mental health outcomes, organised by comparison group. alco, alcohol misuse; anx, anxiety

dep, depression; marij, marijuana use; NCS, National Comorbidity Survey; NCFG, National Survey of Family Growth; suic, suicide.

a pregnancy to term is consistent with literature demonstrating protective effects of pregnancy delivered relative to particular mental health outcomes. For example, with regard to suicide, Gissler et al reported the annual suicide rate for women of reproductive age to be 11.3 per 100000 , whereas the rate was only 5.9 per 100000 in association with birth. ${ }^{34}$ Several other studies conducted in different countries have revealed even lower rates of suicide following birth when compared with women in the general population. ${ }^{44-47}$ More research is needed to examine systematically the specific nature of this protective effect against suicide, to determine the extent to which the protective effect holds for unintended pregnancies delivered, and to examine possible protective effects of childbirth relative to other mental health variables.

When the abortion group was compared with the no pregnancy group and with the unintended pregnancy delivered group, the magnitude of the effects was very close. This finding challenges the generally accepted belief that unintended pregnancy delivered represents the only or most appropriate control group for studies designed to explore the impact of abortion on mental health. Use of a no pregnancy delivered group may be a cleaner control group, since many women experience postpartum depression and/or anxiety following childbirth. From a practical standpoint, a no pregnancy comparison group should be considerably easier to secure than a group of women who deliver an unintended pregnancy.

\section{Future research}

Future studies should explore possible process mechanisms linking abortion to substance misuse and suicidal behaviour, since the strongest effects were detected for these variables. For example, substance misuse and suicidal behaviour may result from efforts to block or avoid any psychological pain associated with the procedure and may be construed as faster, easier remedies for personal suffering than seeking professional help. Women could find it particularly difficult to reach out to others if they experience shame or guilt associated with the abortion. Consistent with the contemporary ethos of evidence-based medicine wherein effective use is made of the best available data from systematic research, firm standards should be articulated for accessing and synthesising information from the published literature for the purpose of training healthcare personnel. The results of this systematic, quantitative review cast serious doubt on the conclusions derived from the recently published traditional reviews described earlier, ${ }^{5-7}$ and suggest that there are in fact some real risks associated with abortion that should be shared with women as they are counselled prior to an abortion decision. 
Healthcare professionals are responsible for educating patients in a manner that reflects the current scientific literature; however, the average practitioner does not generally have the time and expertise to study and attempt to resolve conflicting interpretations of the published research in order to extract the most reliable information. The responsibility therefore rests initially within the research community to set aside personal ideological commitments, objectively examine all high-quality published data, and conduct analyses of the literature that are based on state-ofthe-art data analysis procedures, yielding readily interpretable synopses as has been attempted here. Once this goal is satisfactorily realised, professional organisations will face the challenge of developing efficient protocols for informing practitioners and for streamlining the dissemination of information to the public.

The US Preventive Services Task Force (USPSTF) within the Agency for Healthcare Research and Quality, which is a division of the US Department of Health and Human Services (www. ahrq.gov/clinic/3rduspstf/ratings.htm), has identified basic guidelines for how scientific evidence should be used to inform practice. These are summarised below and are based on an analysis of risks and benefits as established in the scientific literature.

- Level A: Good scientific evidence indicates the benefits of the service substantially outweigh the risks with clinicians advised to discuss the service with eligible patients.

- Level B: Fair scientific evidence indicates the benefits of the service outweigh the risks with clinicians encouraged to discuss the service with eligible patients.

- Level C: At least fair scientific evidence indicating benefits are provided by the service, but the balance between benefits and risks precludes general recommendations. Clinicians are advised to only offer the service if there are special considerations.

- Level D: At least fair scientific evidence indicates the risks of the service outweigh benefits with clinicians advised not to routinely offer the service.

- Level I: Scientific evidence is deficient, poorly done, or conflicting precluding assessment of the risk benefit ratio. Clinicians are advised to convey the uncertainty of evidence surrounding the service to patients.

\section{Putative benefits of abortion}

Procedure benefits of abortion have not been empirically established and the results of the substantial review by Thorp et al described earlier in conjunction with the results of the present quantitative synthesis indicate considerable evidence documenting mental health risks. ${ }^{4}$ Without more research pertaining to possible benefits, the above guidelines are difficult to apply. In one study by Major et $a l^{14}$ the average response of the study respondents reflecting their positive post-abortion emotional reactions (defined as 'happy', 'pleased' or 'satisfied') was 2.24 on a scale of 1 to 5 , with 1 corresponding to 'not at all' and a 5 representing 'a great deal'. The passage of time apparently did not result in more positive emotions, because 2 years after abortion the average rating dropped by a statistically significantly amount to 2.06 . A few additional studies have addressed associations between abortion and educational attainment, income and other outcomes of this nature, which may be construed as indirect indicators of mental health; ${ }^{48,49}$ however, mental health benefits have received scant direct attention in the literature.

Concerns regarding the deficient positive effects literature were echoed in an editorial published in the Psychiatric Bulletin, ${ }^{50}$ in which Fergusson questioned the legitimacy of justifying over $90 \%$ of UK abortions based on the presumption that abortion offers the benefit of reducing mental health risks associated with continuing the pregnancy. Fergusson specifically stated:

Although decisions on whether to proceed with induced abortion are made on the basis of clinical assessments of the extent to which abortion poses a risk to maternal mental health, these clinical assessments are not currently supported by populationlevel evidence showing the provision of abortion reduces mental health risks for women having unwanted pregnancy. ${ }^{50}$

Until sound evidence documenting mental health benefits of abortion is available, clinicians should convey the current state of uncertainty related to benefits of abortion in addition to sharing the most accurate information pertaining to statistically validated risks.

\section{Strengths and limitations of this review}

Motivated by the shortcomings of previous non-quantitative efforts to synthesise and analyse a complex literature prone to biased interpretations, I have attempted in this study to evaluate systematically a wealth of data on the topic of abortion and mental health. The use of inclusion criteria that resulted in incorporation of the largest and strongest studies published in recent years is an obvious strength. However, the review is clearly not exhaustive as only a 15 -year publication window was examined and studies that did not incorporate a comparison group were not analysed. There is a strong need for a quantitative review of literature examining the hundreds of studies that have been conducted on samples of women who obtained abortions without inclusion of a comparison group. As noted previously, the review of literature conducted by the American Psychological Association Task Force confined their examination of this study form to US samples. ${ }^{5}$ Another limitation of my study relates to the lack of uniformity in control variables, demographic characteristics of the samples, length of time between the procedure and the follow-up assessments, and considerable variation in how the outcomes were measured.

It is encouraging to note that methodologically sophisticated studies on the topic of abortion and mental health are being published at a significantly higher rate than ever before. Researchers throughout the world are seeking to understand the experience of induced abortion more fully and are increasingly willing to take on a subject that has been shrouded in political controversy and has not received the scholarly attention it deserves. The latest example is a study based on National Comorbidity Survey - Replication data by Canadian researchers Mota et al. ${ }^{51}$ This 2010 study was published after the analyses reported herein were conducted; however, its results are startlingly similar. Statistically significant associations were observed between abortion history and a wide range of mental health problems after controlling for the experience of interpersonal violence and demographic variables. When compared with women without an abortion history, women with a prior abortion experienced a $61 \%$ increased risk of mood disorders. Abortion was further linked with a $61 \%$ increased risk of social phobia, and increased the risk of suicide ideation by $59 \%$. In the realm of substance misuse, the abortion-related increased risks for alcohol misuse, alcohol dependence, drug misuse, drug dependence and any substance use disorder were 261\%, 142\%, 313\%, 287\% and $280 \%$ respectively. Population-attributable risk percentages were likewise similar, ranging from $5.8 \%$ to $24.7 \% .^{51}$

\section{Concluding remarks}

This review was undertaken in an effort to produce an unbiased, quantitative analysis of the best available evidence addressing abortion as one risk factor among many others that may increase the likelihood of mental health problems. The composite results reported herein indicate that abortion is a statistically validated risk factor for the development of various psychological disorders. However, when the independent variable cannot be ethically manipulated, as is the case with abortion history, definitive causal 
conclusions are precluded from both individual studies and from a quantitative synthesis such as this one. Although an answer to the causal question is not readily discerned based on the data available, as more prospective studies with numerous controls are being published, indirect evidence for a causal connection is beginning to emerge.

Priscilla K. Coleman, Human Development and Family Studies, 16 D FCS Building, Bowling Green State University, Bowling Green, Ohio 43402, USA. Email: pcolema@bgnet.bgsu.edu

First received 1 Jan 2010, final revision 5 Oct 2010, accepted 21 Mar 2011

\section{References}

1 Bradshaw Z, Slade $P$. The effects of induced abortion on emotional experiences and relationships: a critical review of the literature. Clin Psychol Rev 2003; 23: 929-58.

2 Coleman PK. Induced abortion and increased risk of substance use: a review of the evidence. Curr Women's Health Rev 2005; 1: 21-34.

3 Coleman PK, Reardon DC, Strahan TS, Cougle JR. The psychology of abortion: a review and suggestions for future research. Psychol Health 2005; 20: 237-71.

4 Thorp J, Hartman K, Shadigan E. Long-term physical and psychological health consequences of induced abortion: review of the evidence. Obstet Gynecol Surv 2003; 58: 67-79.

5 American Psychological Association Task Force on Mental Health and Abortion. Report of the American Psychological Association Task Force on Mental Health and Abortion. APA, 2008.

6 Charles VE, Polis CB, Sridhara SK, Blum RW. Abortion and long-term menta health outcomes: a systematic review of the evidence. Contraception 2008; 78: 436-50.

7 Robinson GE, Stotland NL, Russo NF, Lang JA, Occhiogrosso M. Is there an 'abortion trauma syndrome'? Critiquing the evidence. Harv Rev Psychiatry 2009; 17: 268-90.

8 Kost K, Forrest JD. Intention status of US births in 1988: differences by mothers' socioeconomic and demographic characteristics. Fam Plann Perspect 1995; 27: 11-7.

9 Squires S. Most pregnancies unplanned or unwanted, study says. Washington Post 9 May 1995; 11: 7.

10 Coleman PK. Resolution of unwanted pregnancy during adolescence through abortion versus childbirth: individual and family predictors and consequences. J Youth Adolesc 2006; 35: 903-11.

11 Henshaw R, Naji S, Russell I, Templeton A. Psychological responses following medical abortion (using mifepristone and gemeprost) and surgical vacuum aspiration: a patient-centered, partially randomized prospective study. Acta Obstet Gynec Scand 1994; 73: 812-8.

12 Lauzon P, Roger-Achim D, Achim A, Boyer R. Emotional distress among couples involved in first trimester abortions. Can Fam Physician 2000; 46: 2033-40.

13 Lyndon J, Dunkel-Schetter C, Cohan CL, Pierce T. Pregnancy decision making as a significant life event: a commitment approach. J Pers Soc Psychol 1996; 71: 141-51.

14 Major B, Cozzarelli C, Cooper ML, Zubek J, Richards C, Wilhite M, et al. Psychological responses of women after first trimester abortion. Arch Gen Psychiatry 2000; 57: 777-84.

15 Major B, Cozzarelli C, Sciacchitano AM, Cooper ML, Testa M, Mueller PM. Perceived social support, self-efficacy, and adjustment to abortion. J Pers Soc Psychol 1990; 59: 186-97.

16 Miller WB. An empirical study of the psychological antecedents and consequences of induced abortion. J Soc Issues 1992; 48: 67-93.

17 Miller WB, Pasta DJ, Dean CL. Testing a model of the psychological consequences of abortion. In The New Civil War: The Psychology, Culture, and Politics of Abortion (eds LJ Beckman, SM Harvey): pp. 235-67. American Psychological Association, 1998.

18 Reardon DC, Coleman PK. Relative treatment rates for sleep disorders following abortion and childbirth: a prospective record-based study. Sleep 2006; 29: 105-6.

19 Slade P, Heke S, Fletcher J, Stewart PA. comparison of medical and surgical methods of termination of pregnancy: choice, psychological consequences, and satisfaction with care. Br J Obstet Gynaecol 1998; 105: 1288-95.

20 Coleman PK, coyle CT, Shuping M, Rue VM. Induced abortion and anxiety, mood, and substance abuse disorders: isolating the effects of abortion in the National Comorbidity Survey. J Psychiatr Res 2009; 43: 770-6.

21 Dingle K, Alati R, Clavarino A, Najman JM, Williams GM. Pregnancy loss and psychiatric disorders in young women: an Australian birth cohort study. Br J Psychiatry 2008; 193: 455-60.
22 Fergusson DM, Horwood L, Boden JM. Abortion and mental health disorders: evidence from a 30-year longitudinal study. Br J Psychiatry 2008; 193: 444-51.

23 Fergusson DM, Horwood $\sqcup$, Ridder EM. Abortion in young women and subsequent mental health. J Child Psychol Psychiatry 2006; 47: 16-24.

24 Pedersen W. Childbirth, abortion and subsequent substance use in young women: a population-based longitudinal study. Addiction 2007; 102: 1971-8.

25 Lipsey MW. Identifying interesting variables and analysis opportunities. In The Handbook of Research Synthesis and Meta-Analysis, 2nd edn (eds H Cooper, LV Hedges, JC Valentine): 147-58. Russell Sage Foundation, 2009.

26 Coleman PK, Reardon DC, Cougle J. Substance use among pregnant women in the context of previous reproductive loss and desire for current pregnancy. Br J Health Psychol 2005; 10: 255-68.

27 Steinberg JR, Russo NF. Abortion and anxiety: what's the relationship? SOC Sci Med 2008; 67: 238-52.

28 Coleman PK, Maxey DC, Spence M, Nixon C. The choice to abort among mothers living under ecologically deprived conditions: predictors and consequences. Int J Ment Health Addiction 2009; 7: 405-22.

29 coleman PK, Reardon DC, Rue V, Cougle J. History of induced abortion in relation to substance use during subsequent pregnancies carried to term. Am J Obstet Gynecol 2002; 187: 1673-8.

30 Coleman PK, Reardon DC, Rue V, Cougle J. State-funded abortions vs. deliveries: a comparison of outpatient mental health claims over four years. Am J Orthopsychiatry 2002; 72: 141-52.

31 Cougle J, Reardon DC, Coleman PK. Depression associated with abortion and childbirth: a long-term analysis of the NLSY cohort. Med Sci Monit 2003; 9: CR105-12.

32 Cougle J, Reardon DC, Coleman PK, Rue VM. Generalized anxiety associated with unintended pregnancy: a cohort study of the 1995 National Survey of Family Growth. J Anxiety Disord 2005; 19: 137-42.

33 Gilchrist AC, Hannaford PC, Frank P, Kay CR. Termination of pregnancy and psychiatric morbidity. Br J Psychiatry 1995; 167: 243-8.

34 Gissler M, Hemminki E, Lonnqvist J. Suicides after pregnancy in Finland, 1987-94: register linkage study. BMJ 1996; 313: 1431-4.

35 Pedersen W. Abortion and depression: a population-based longitudinal study of young women. Scand J Public Health 2008; 36: 424-8.

36 Reardon DC, Cougle J. Depression and unintended pregnancy in the National Longitudinal Survey of Youth: a cohort study. BMJ 2002; 324: 151-2.

37 Reardon DC, Cougle J, Ney PG, Scheuren F, Coleman PK, Strahan TW. Deaths associated with delivery and abortion among California Medicaid patients: a record linkage study. South Med J 2002; 95: 834-41.

38 Reardon DC, Cougle J, Rue VM, Shuping M, Coleman PK, Ney PG. Psychiatric admissions of low-income women following abortion and childbirth. CMAJ 2003; 168: 1253-6.

39 Reardon DC, Coleman PK, Cougle J. Substance use associated with prior history of abortion and unintended birth: a national cross sectional cohort study. Am J Drug Alcohol Abuse 2004; 26: 369-83.

40 Rees DI, Sabia JJ. The relationship between abortion and depression: new evidence from the Fragile Families and Child Wellbeing Study. Med Sci Monit 2007; 13: 430-6.

41 Schmiege S, Russo NF. Depression and unwanted first pregnancy: longitudinal cohort study. BMJ 2005; 331: 1303.

42 Taft AJ, Watson LF. Depression and termination of pregnancy (induced abortion) in a national cohort of young Australian women: the confounding effect of women's experience of violence. BMC Public Health 2008; 8: 75.

43 Borenstein M, Hedges LV, Higgins JPT, Rothstein HR. Introduction to MetaAnalysis. Wiley, 2009.

44 Appleby L. Suicide during pregnancy and in the first postnatal year. BMJ 1991; 302: 137-40.

45 Kleiner GJ, Greston WM (eds). Suicide in Pregnancy. John Wright, 1984

46 Lindahl V, Pearson JL, Colpe L. Prevalence of suicidality during pregnancy and the postpartum. Arch Womens Ment Health 2005; 8: 77-87.

47 Schiff MA, Grossman DC. Adverse perinatal outcomes and risk of postpartum suicide attempt in Washington State, 1987-2001. Pediatrics 2006; 118: e669-75.

48 Bailey PE, Bruno ZV, Bezerra MF, Queiroz J, Oliveira CM, Chen-Mok M. Adolescent pregnancy 1 year later: the effects of abortion vs. motherhood in Northeast Brazil. J Adolesc Health 2001; 29: 223-32.

49 Fergusson DM, Boden JM, Horwood L. Abortion among young women and subsequent life outcomes. Perspect Sex Reprod Health 2007; 39: 6-12.

50 Fergusson DM. Abortion and mental health. Psychiatr Bull 2008; 32: 321-4.

51 Mota NP, Burnett M, Sareen J. Associations between abortion, mental disorders, and suicidal behaviour in a nationally representative sample. Can J Psychiatry 2010; 55: 239-47. 